\title{
Triglyceride/High-Density Lipoprotein Cholesterol Ratio is Associated with the Mortality of COVID-19: A Retrospective Study in China
}

\author{
Fei Peng', Si Lei', Quan Zhang', Yanjun Zhong², Shangjie Wu' \\ 'Department of Respiratory Medicine, The Second Xiangya Hospital of Central South University, Changsha, People's Republic of China; ${ }^{2}$ Department \\ of Critical Care Medicine, The Second Xiangya Hospital of Central South University, Changsha, People's Republic of China \\ Correspondence: Yanjun Zhong, Department of Critical Care Medicine, The Second Xiangya Hospital of Central South University, No. I39 Middle \\ Renmin Road, Changsha, 4I00II, People’s Republic of China, Email zhongyanjun@csu.edu.cn \\ Shangjie Wu, Department of Respiratory Medicine, The Second Xiangya Hospital of Central South University, No. I39 Middle Renmin Road, \\ Changsha, 4I00II, People's Republic of China, Email wushangjie@csu.edu.cn
}

Background: Triglyceride to high density lipoprotein cholesterol ratio (TG/HDL-c) is crucial when researching metabolic and vascular diseases, and its involvement in COVID-19 was sparsely elaborated on. The purpose of the study was to explore the inflammatory associations between the TG/HDL-c ratio and COVID-19 prognosis.

Methods: A total of 262 COVID-19 patients consisting of 244 survivors and 18 non-survivors were retrospectively investigated. The clinical features and baseline hematological parameters were recorded and analyzed. The receiver operating characteristic curve (ROC) was used to explore the role of TG/HDL-c in predicting the mortality of COVID-19, the Spearman's rank correlation coefficients were used to measure the correlation between TG/HDL-c and inflammatory indicators, and the Kaplan-Meier (KM) curve was used to estimate the survival of COVID-19 patients with high and low TG/HDL-c ratio. Logistic regression analyses were performed to investigate the role of TG/HDL-c ratio on mortality of COVID-19 with no underlying diseases.

Results: Compared with the survivors, the non-survivors of COVID-19 had significantly higher levels of white blood cells (4.7 vs $13.0 \times$ $\left.10^{9} / \mathrm{L} ; P<0.001\right)$, neutrophils $\left(3.0\right.$ vs $\left.11.6 \times 10^{9} / \mathrm{L} ; P<0.001\right)$, C-reactive proteins $(15.7 \mathrm{vs} 76.7 \mathrm{mg} / \mathrm{L} ; P<0.001)$ and TG/HDL-c ratio (1.4 vs $2.5 ; P=0.001)$. The ROC curve [area under the curve (AUC), $0.731 ; 95 \%$ confidence interval $(C I), 0.609-0.853 ; P=0.001$ ] suggested that the TG/HDL-c ratio could predict the mortality of COVID-19. The TG/HDL-c ratio was positively correlated with white blood cells $(r=0.255, P<0.001)$, neutrophils $(r=0.243, P<0.001)$ and $\mathrm{C}$-reactive proteins $(r=0.170, P<0.006)$. Patients with high TG/ HDL-c ratio showed a worse survival compared with those with low TG/HDL-c ratio (Log rank $P=0.003$ ). Moreover, TG/HDL-c ratio was an independent factor in predicting the mortality of COVID-19 patients with no underlying diseases.

Conclusion: Our study demonstrated that TG/HDL-c ratio might potentially be a predictive marker for mortality in COVID-19 patients.

Keywords: COVID-19, triglyceride, high density lipoprotein cholesterol, inflammation, viral infection, triglyceride-to-high-density lipoprotein cholesterol ratio, mortality

\section{Introduction}

As is generally known, Coronavirus disease 2019 (COVID-19), caused by severe acute respiratory syndrome coronavirus 2 (SARS-CoV-2), has already become a serious threat to the global public health system. ${ }^{1}$ It is more contagious than SARS and Middle East respiratory syndrome, both of which are from the same family of RNA virus as COVID-19. ${ }^{2}$ Despite a relatively higher incidence of mild cases, once it gets worse, severe cases can progress rapidly, culminating in respiratory failure, septic shock or a fatal outcome. In this regard, it is of great significance to identify relevant risk factors for COVID-19 progression. 
Undoubtedly, dyslipidemia is strongly associated with obesity and obesity-related disorders, and is commonly found in patients with stroke, insulin resistance, metabolic syndrome and cardiovascular diseases. ${ }^{3-6}$ Over recent years, obese state has been characterized by oxidative stress. ${ }^{7}$ It was reported that hypertrophied adipocytes, which promoted the dysfunction of adipose tissue, could produce a high level of reactive oxygen species (ROS), participating in many metabolic signaling pathways, such as insulin sensitivity, inflammation, and endothelial dysfunction. Meanwhile, inflammation could lead to a rapid spike in levels of ROS, which established a feedback-loop between inflammation and oxidative stress. ${ }^{7}$ Increase in triglyceride (TG) and decrease in high density lipoprotein cholesterol (HDL-c) may be caused by inflammatory cytokines, and it has been suggested that triglyceride to high density lipoprotein cholesterol ratio (TG/HDL-c ratio), a simple, non-invasive, and convenient measurement indicator, could integrate predictive risks of two parameters into a single risk factor and showed better predictive value than TG and HDL-c alone in atherosclerotic disturbances, ${ }^{8}$ and our results were consistent with the study (in Supplementary Figures 1 and 2 ). Klisic et al demonstrated that TG/HDL-c had positive correlation with high sensitivity C-reactive protein (hsCRP) and inflammatory biomarker cystatin $\mathrm{C}$ in adolescent girls. ${ }^{9}$ Idiopathic pulmonary arterial hypertension (IPAH) patients with higher TG/ HDL-c ratio were prone to suffer from systemic inflammation. ${ }^{10}$ As for COVID-19, it was reported that TG/HDL-c ratio was a determining of cardiovascular risk in COVID-19; ${ }^{11}$ low HDL and high triglyceride could predict the severity of COVID-19; ${ }^{12}$ TG/HDL-c ratio was correlated with indicators for severity of COVID-19, such as levels of lactate dehydrogenase, National Early Warning Score 2 (NEWS 2), and quick sequential organ failure assessment, which could be a biomarker for poor prognosis with requirement of invasive mechanical ventilation. ${ }^{13}$ Nevertheless, there are few studies with respect to the inflammatory correlation between TG/HDL-c ratio and the prognosis of COVID-19, which reminds to be further elaborated on. In view of the current status of COVID-19 pandemic and the potential role of TG/ HDL-c ratio mentioned above, this study was performed with the purpose to clarify the inflammatory relationship of TG/HDL-c ratio and the mortality of COVID-19 patients, so as to provide potential reference for monitoring and preventing COVID-19 progression.

\section{Materials and Methods}

\section{Study Design and Participants}

The study was conducted by the Second Xiangya Hospital of Central South University in March 2021, and Ethic approval was obtained from the Second Xiangya Hospital of Central South University (No.2020001). 622 COVID-19 patients were all adults who were confirmed by real-time polymerase chain reactions from Tongji Medical College of Huazhong University of Science and Technology, Wuhan, and Changsha Public Health Treatment Center, China, by March 26th 2020. ${ }^{14}$ Among the patients, 360 of them whose lipids test was missing were excluded. Thus, 262 patients were enrolled in the study (Figure 1). Furthermore, enrolled patients were divided into survivor group and non-survivor group based on their survival status. To avoid the impact of underlying diseases on COVID-19, 88 patients with hypertension, cardiovascular disease, diabetes, COPD, chronic bronchitis, cerebrovascular disease, or cancer were excluded, and further studies were conducted to investigate the role of TG/HDL-c ratio in COVID-19 with no underlying diseases.

\section{Data Collection}

All medical records of the enrolled patients were carefully collected and reviewed by two members of our team. We recorded the demographics, baseline characteristics, and blood test parameters of these patients on admission. The medical records with incomplete information were excluded. The primary end point in this study was the occurrence of death among patients with COVID-19. 


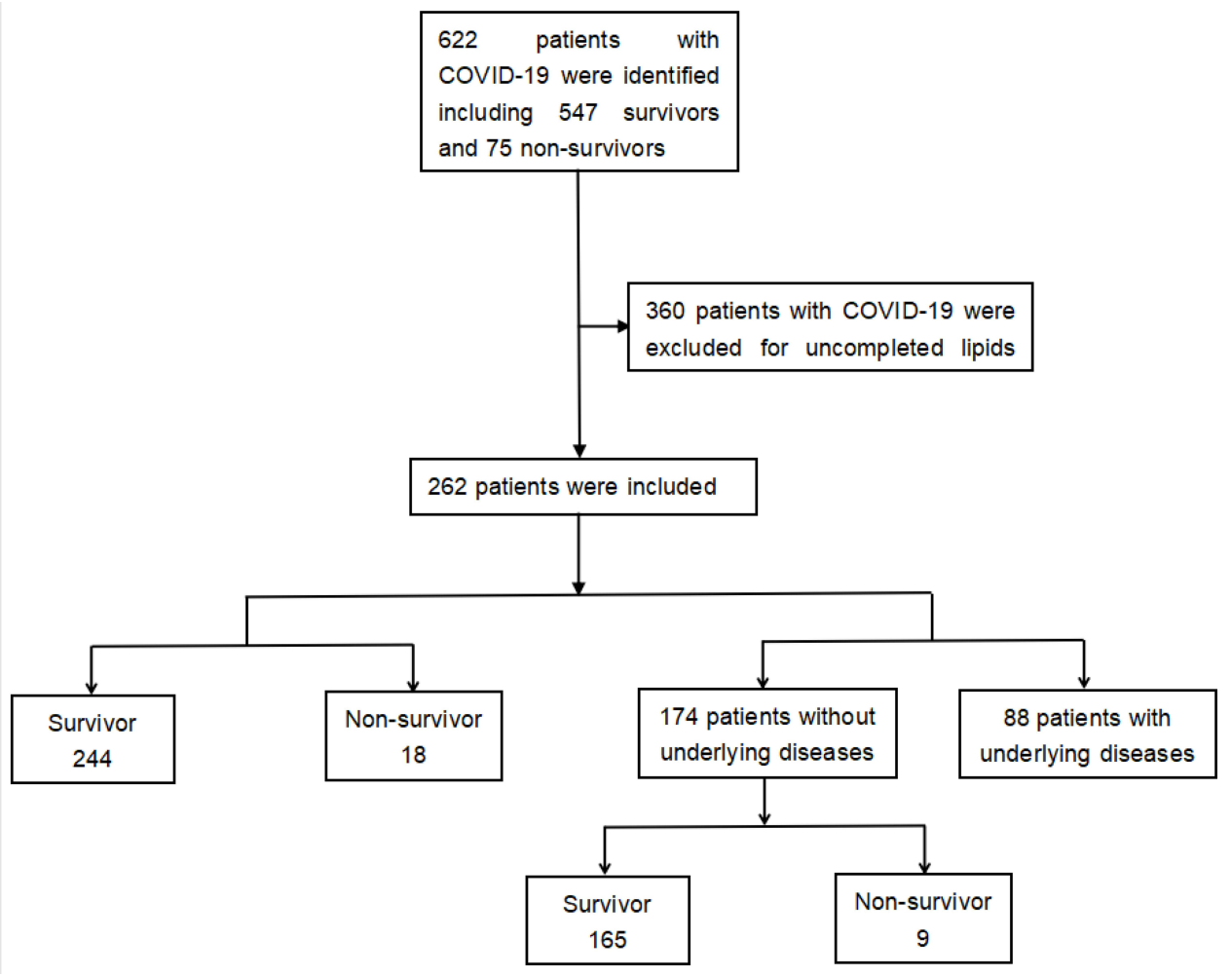

Figure I Flow chart of patient enrollment. 262 patients were enrolled in the study.

\section{Definition and Endpoints}

Low TG/HDL-c ratio is defined as below the cutoff value of receiver operating characteristic curve (ROC) analysis for the role of TG/HDL-c in predicting the mortality of COVID-19, and high TG/HDL-c ratio is defined as above or equal to the cutoff value of ROC analysis for the role of TG/HDL-c in predicting the mortality of COVID-19. The endpoint was the mortality of patients with COVID-19 who died during hospitalization.

\section{Statistical Analysis}

Considering the non-normal distribution of all continuous variables, these data were presented as Median with interquartile range, and Mann-Whitney test was used to compare the difference between groups. All categorical variables were described as number and percentages, and Fisher's exact test (or $\chi^{2}$ test) was used for data analysis. To detect which variables best predicted the final outcome for COVID-19 patients, we performed univariate and multivariate logistic regression analyses. To evaluate how variables can predict COVID-19 prognoses, the ROC were calculated. The Spearman's rank correlation coefficients were used to measure the correlation between two variables. The KaplanMeier (KM) curve with Log rank tests were used to estimate the survival of COVID-19 patients with high and low TG/ HDL-c ratios. IBM SPSS version 26 software (IBM ${ }^{\mathbb{B}}$ SPSS, Chicago, IL, USA) was conducted during all analyses.

\section{Results}

The enrolled patients were separated into survivors and non-survivors based on clinical outcomes, and demographics and baseline characteristics for each group are provided in Table 1. A total of 262 patients aged above 18 were admitted to the study, which included 244 survivors and 18 non-survivors. Based on their age, patients were further divided into three groups, ie, $\geq 65$ years $(21.4 \%), 45$ years $\leq$ age $<65$ years $(36.6 \%)$, and $<45$ years $(42.0 \%)$. Comparing the survivors with the non-survivors, there were significant differences in the ages and comorbidities with cancer $(P<0.05)$. 
Table I Demographics and Baseline Characteristics of Survivor and Non-Survivor of COVID-I9 Patients

\begin{tabular}{|c|c|c|c|c|}
\hline & No. $(\%)$ Total $(n=262)$ & Survivor $(n=244)$ & Non-Survivor $(\mathrm{n}=18)$ & $P$ value \\
\hline Age, \% & & & & $<0.001$ \\
\hline$\geq 65 y$ & $56(21.4)$ & $45(18.4)$ & II (6I.I) & \\
\hline $45 \leq$ age $<65$ & $96(36.6)$ & $89(36.5)$ & 7 (38.9) & \\
\hline$<45 y$ & $110(42.0)$ & $110(45.1)$ & $0(0.0)$ & \\
\hline Gender, \% & & & & 0.774 \\
\hline Male & $137(52.3)$ & $127(52.0)$ & $10(55.6)$ & \\
\hline Female & $125(47.7)$ & $117(48.0)$ & $8(44.4)$ & \\
\hline \multicolumn{5}{|l|}{ Symptoms } \\
\hline Fever, \% & I8I (78.6) & $165(67.6)$ & $16(88.9)$ & 0.060 \\
\hline Cough, \% & $212(80.9)$ & 197 (80.7) & $15(83.3)$ & 0.787 \\
\hline Myalgia, \% & $26(9.9)$ & $25(10.2)$ & I (5.6) & 0.521 \\
\hline Fatigue, $\%$ & II $4(43.5)$ & $109(44.7)$ & $5(27.8)$ & 0.164 \\
\hline Headache, \% & $33(12.6)$ & $32(13.1)$ & I (5.6) & 0.352 \\
\hline Diarrhoea, \% & $58(22.1)$ & $54(22.1)$ & $4(22.2)$ & 0.993 \\
\hline Abdominal pain, $\%$ & $7(2.7)$ & $7(2.9)$ & $0(0.0)$ & 0.467 \\
\hline Shortness of breath, $\%$ & $90(34.3)$ & $83(34.0)$ & 7 (38.9) & 0.675 \\
\hline \multicolumn{5}{|l|}{ Comorbidities } \\
\hline Hypertension, \% & $53(20.2)$ & $48(19.7)$ & $5(27.8)$ & 0.410 \\
\hline Cardiovascular disease, \% & $18(6.9)$ & $15(6.1)$ & $3(16.7)$ & 0.089 \\
\hline Diabetes, \% & $25(9.5)$ & $23(9.4)$ & $2(11.1)$ & 0.815 \\
\hline COPD, \% & $\mathrm{I}(0.0)$ & I $(0.0)$ & $0(0.0)$ & 0.786 \\
\hline Chronic bronchitis, \% & $20(7.6)$ & $20(8.1)$ & $0(0.0)$ & 0.527 \\
\hline Cerebrovascular disease, \% & $9(3.4)$ & $8(3.2)$ & I (5.5) & 0.609 \\
\hline Cancer, \% & $5(1.9)$ & $2(1.0)$ & $3(16.7)$ & $<0.001$ \\
\hline
\end{tabular}

Notes: $P$ values indicate differences between survivor and non-survivor of COVID-19 patients. $P<0.05$ was considered statistically significant (marked in bold). Abbreviations: COVID-19, Coronavirus disease 2019; COPD, chronic obstructive pulmonary disease.

The non-survivor COVID-19 patients had significantly higher white blood cells (WBC) $\left(4.7\right.$ vs $13.0 \times 10^{9} / \mathrm{L}$; $P<0.001$ ), neutrophils (3.0 vs $\left.11.6 \times 10^{9} / \mathrm{L} ; P<0.001\right)$, C-reactive proteins (CRP) $(15.7 \mathrm{vs} 76.7 \mathrm{mg} / \mathrm{L} ; P<0.001)$, and TG/HDL-c ratio (1.4 vs $2.5 ; P=0.001)$ levels than survivors, but the levels of lymphocytes $\left(1.1 \mathrm{vs} 0.6 \times 10^{9} / \mathrm{L} ; P<0.001\right)$, and low density lipoprotein cholesterol-to-high-density lipoprotein cholesterol ratio (LDL-c/HDL-c) (3.2 vs 2.2; $P=0.010$ ) were lower in non-survivors when compared with survivors (Table 2).

The mortality of COVID-19 patients was associated with age [odds ratio $(O R)=1.108 ; 95 \% C I, 1.060-1.159 ; P<$ $0.001]$, cancer $(O R=24.200 ; 95 \% C I, 3.754-156.023 ; P=0.001)$, WBC $(O R=1.451 ; 95 \%$ CI, $1.267-1.661 ; P<0.001)$,

Table 2 Comparison of Laboratory Parameters Between the Survivor and Non-Survivor of COVID19 Patients

\begin{tabular}{|l|l|l|l|}
\hline & Survivor & Non-Survivor & P value \\
\hline WBC, $\times 10^{9} / \mathrm{L}$ & $4.7(3.6-6.0)$ & $13.0(7.8-17.8)$ & $<0.001$ \\
Lymphocytes, $\times 10^{9} / \mathrm{L}$ & $1.1(0.8-1.5)$ & $0.6(0.4-0.7)$ & $<0.00 \mathrm{I}$ \\
Neutrophils, $\times 10^{9} / \mathrm{L}$ & $3.0(2.2-3.8)$ & $11.6(6.9-16.6)$ & $<0.00 \mathrm{I}$ \\
$\mathrm{CRP}, \mathrm{mg} / \mathrm{L}$ & $15.7(4.5-35.3)$ & $76.7(36.8-229.0)$ & $<0.00 \mathrm{I}$ \\
TC/HDL-c ratio & $4.5(3.8-5.6)$ & $5.0(3.4-5.6)$ & 0.899 \\
LDL-c/HDL-c ratio & $3.2(2.5-4.1)$ & $2.2(1.4-3.4)$ & $\mathbf{0 . 0 1 0}$ \\
TG/HDL-c ratio & $1.4(0.9-2.1)$ & $2.5(1.5-4.8)$ & $\mathbf{0 . 0 0 I}$ \\
\hline
\end{tabular}

Notes: $P$ values indicate differences between survivor and non-survivor of COVID-19 patients. $P<0.05$ was considered statistically significant (marked in bold).

Abbreviations: COVID-19, Coronavirus disease 2019; WBC, White blood cells; CRP, C-reactive proteins; TC/HDL-c ratio, total cholesterol-to-high-density lipoprotein cholesterol ratio; LDL-c/HDL-c ratio, low-density lipoprotein cholesterol-to-high-density lipoprotein cholesterol ratio; TG/HDL-c ratio, triglyceride-to-high-density lipoprotein cholesterol ratio. 
Table 3 Univariate Analysis of Risk Factors Related to the Mortality of COVID-19 Patients

\begin{tabular}{|l|l|l|}
\hline Variables & Odds Ratio $(95 \% \mathbf{C I})$ & $P$ value \\
\hline Age & $1.108(1.060-1.159)$ & $<0.00 I$ \\
Cancer & $24.200(3.754-156.023)$ & $0.00 I$ \\
WBC & $1.45 I(1.267-1.66 \mathrm{I})$ & $<0.00 \mathrm{I}$ \\
Lymphocytes & $0.006(0.00 \mathrm{I}-0.059)$ & $<0.00 \mathrm{I}$ \\
Neutrophils & $1.493(1.294-1.724)$ & $<0.00 \mathrm{I}$ \\
CRP & $1.023(1.014-1.032)$ & $<0.00 \mathrm{I}$ \\
LDL-c/HDL-c ratio & $0.55 \mathrm{I}(0.327-0.927)$ & 0.025 \\
TG/HDL-c ratio & $1.291(1.066-1.564)$ & 0.009 \\
\hline
\end{tabular}

Notes: $P$ values indicate differences between the survivor and non-survivor COVID-19 patients. $P<$ 0.05 was considered statistically significant.

Abbreviations: COVID-19, Coronavirus disease 19; Cl, confidence interval; WBC, White blood cells; CRP, C-reactive proteins; LDL-c/HDL-c ratio, low-density lipoprotein cholesterol-to-highdensity lipoprotein cholesterol ratio; TG/HDL-c ratio, triglyceride-to-high-density lipoprotein cholesterol ratio.

lymphocytes $(O R=0.006 ; 95 \% C I, 0.001-0.059 ; P<0.001)$, neutrophils $(O R=1.493 ; 95 \% C I, 1.294-1.724 ; P<0.001)$, CRP $(O R=1.023 ; 95 \% C I, 1.014-1.032 ; P<0.001)$, LDL-c/HDL-c ratio $(O R=0.551 ; 95 \% C I, 0.327-0.927 ; P=$ $0.025)$, and TG/HDL-c ratio $(O R=1.291 ; 95 \% C I, 1.066-1.564 ; P=0.009)$ (Table 3). However, only TG/HDL-c ratio $(O R=1.730 ; 95 \% C I, 1.044-2.866 ; P=0.033)$ and cancer $(O R=44.973 ; 95 \% C I, 2.059-982.524 ; P=0.016)$ were the independent risk factors affected mortality in COVID-19 patients (Table 4).

ROC analysis indicated that the TG/HDL-c ratio was able to significantly predict death in COVID-19 patients $(P=$ 0.001 ) with the cutoff value of 1.744 , and the area under the curve (AUC) was 0.731 (95\% CI, 0.609-0.853) with sensitivity and specificity of 0.722 and 0.656 , respectively (Figure 2). Bivariate correlations indicated that TG/HDL-c ratio was positively correlated with WBC $(r=0.255, P<0.001)$, neutrophils $(r=0.243, P<0.001)$ and CRP $(r=0.170$, $P<0.006)$, but no significant correlations existed between TG/HDL-c ratio and lymphocytes $(r=-0.004, P=0.949)$ (Figure 3). According to the cutoff value 1.744, we divided the patients' TG/HDL-c ratio into high and low TG/HDL-c ratio, and found that high TG/HDL-c ratio showed a worse survival compared with those with low TG/HDL-c ratio (Log rank $P=0.003$ ) (Figure 4).

Eighty-eight patients with hypertension, cardiovascular disease, diabetes, COPD, chronic bronchitis, cerebrovascular disease, or cancer were excluded, the remaining patients were separated into survivors and non-survivors based on demographics and baseline characteristics in Table 5. A total of 174 patients consisting of 165 survivors and 9 non-survivors were included in the study. Age was significant difference comparing the survivors with the non-survivors $(P<0.05)$.

The non-survivor COVID-19 patients with no underlying diseases had significantly higher WBC $\left(4.6 \mathrm{vs} 12.6 \times 10^{9} / \mathrm{L}\right.$; $P<0.001)$, neutrophils $\left(2.9\right.$ vs $\left.11.4 \times 10^{9} / \mathrm{L} ; P<0.001\right)$, CRP $(12.7$ vs $70.1 \mathrm{mg} / \mathrm{L} ; P<0.001)$, and TG/HDL-c ratio $(1.3$ vs

Table 4 Multivariate Analysis of Risk Factors Related to the Mortality of COVID-19 Patients

\begin{tabular}{|l|l|l|l|l|l|l|}
\hline & B & SE & Wald & P & OR & $\mathbf{9 5 \%}$ Cl \\
\hline Age & 0.050 & 0.039 & 1.703 & 0.192 & 1.052 & $0.975-I .134$ \\
Cancer & 3.806 & 1.574 & 5.851 & $\mathbf{0 . 0 1 6}$ & 44.973 & $2.059-982.524$ \\
WBC & 0.919 & 1.322 & 0.484 & 0.487 & 0.399 & $0.030-5.319$ \\
Lymphocytes & 3.388 & 2.375 & 2.035 & 0.154 & 0.034 & $0.000-3.550$ \\
Neutrophils & 1.055 & 1.358 & 0.604 & 0.437 & 2.872 & $0.201-41.096$ \\
CRP & 0.007 & 0.007 & 1.113 & 0.292 & 1.007 & $0.994-1.021$ \\
LDL-c/HDL-c ratio & 0.323 & 0.307 & 1.111 & 0.292 & 0.724 & $0.397-1.321$ \\
TG/HDL-c ratio & 0.548 & 0.258 & 4.522 & $\mathbf{0 . 0 3 3}$ & 1.730 & $1.044-2.866$ \\
\hline
\end{tabular}

Notes: $P$ values indicate differences between the survivor and non-survivor COVID- 19 patients. $P<0.05$ was considered statistically significant (marked in bold). Abbreviations: COVID-19, Coronavirus disease 19; SE, standard error; OR, odds ratio; CI, confidence interval; WBC, White blood cells; CRP, C-reactive proteins; LDL-c/ HDL-c ratio, low-density lipoprotein cholesterol-to-high-density lipoprotein cholesterol ratio; TG/HDL-c ratio, triglyceride-to-high-density lipoprotein cholesterol ratio. 


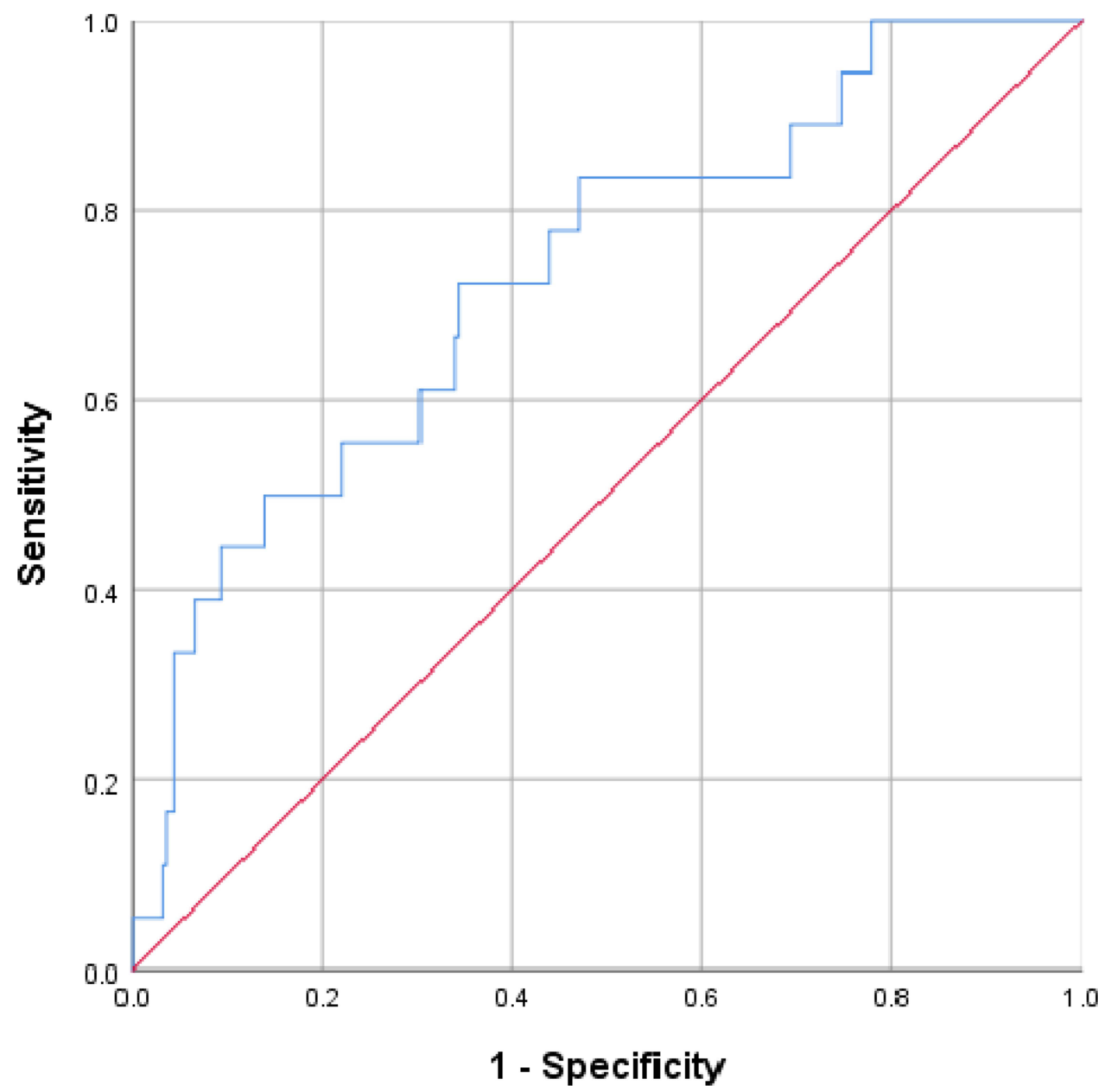

Figure 2 Receiver operating characteristic curve of TG/HDL-c ratio for predicting the mortality of COVID-19. The TG/HDL-c ratio was able to significantly predict death in COVID-19 patients $(P=0.00 \mathrm{I})$ with the area under the curve of $0.73 \mathrm{I}(95 \% \mathrm{Cl}, 0.609-0.853)$.

1.9; $P=0.001)$ levels than survivors, but the levels of lymphocytes $\left(1.2\right.$ vs $\left.0.6 \times 10^{9} / \mathrm{L} ; P<0.001\right)$, and low density lipoprotein cholesterol-to-high-density lipoprotein cholesterol ratio (LDL-c/HDL-c) (3.2 vs $2.2 ; P=0.010)$ were lower in non-survivors when compared with survivors (Table 6).

The mortality of COVID-19 patients with no underlying diseases was associated with WBC $(P<0.001)$, lymphocytes $(P<0.001)$, neutrophils $(P<0.001)$, CRP $(P=0.002)$, and TG/HDL-c ratio $(P=0.008)$. However, only TG/HDL-c ratio $(O R=1.744 ; 95 \% C I, 1.091-2.786 ; P=0.020)$ was the independent risk factor (Table 7).

\section{Discussion}

Our study reported three findings primarily. Firstly, there was a rise in TG/HDL-c ratio of the non-survivors when compared with that of the survivors. Secondly, TG/HDL-c ratio levels in patients on admission was positively correlated with inflammatory indicators, such as WBC, neutrophils, and CRP. Finally, TG/HDL-c ratio in patients on admission might be able to predict and measure COVID-19 mortality.

It has been verified that TG levels might increase during infection and inflammation. ${ }^{15,16}$ Inflammatory cytokines might contribute to TG synthesis and reduce TG hydrolysis under septic conditions, ${ }^{17}$ and could also increase the angiopoietin-like protein 4 expressions that might further suppress TG-rich lipoprotein metabolism. ${ }^{18}$ In addition, tumor necrosis factor (TNF)- $\alpha$ was reported to produce a rapid rise in TG concentration through increasing hepatic production. ${ }^{18}$ Without doubt, due to social isolation and long period of stay at home, people were prone to have an imbalanced diet and be less active, which might further worsen their metabolic and lipid profiles to induce 

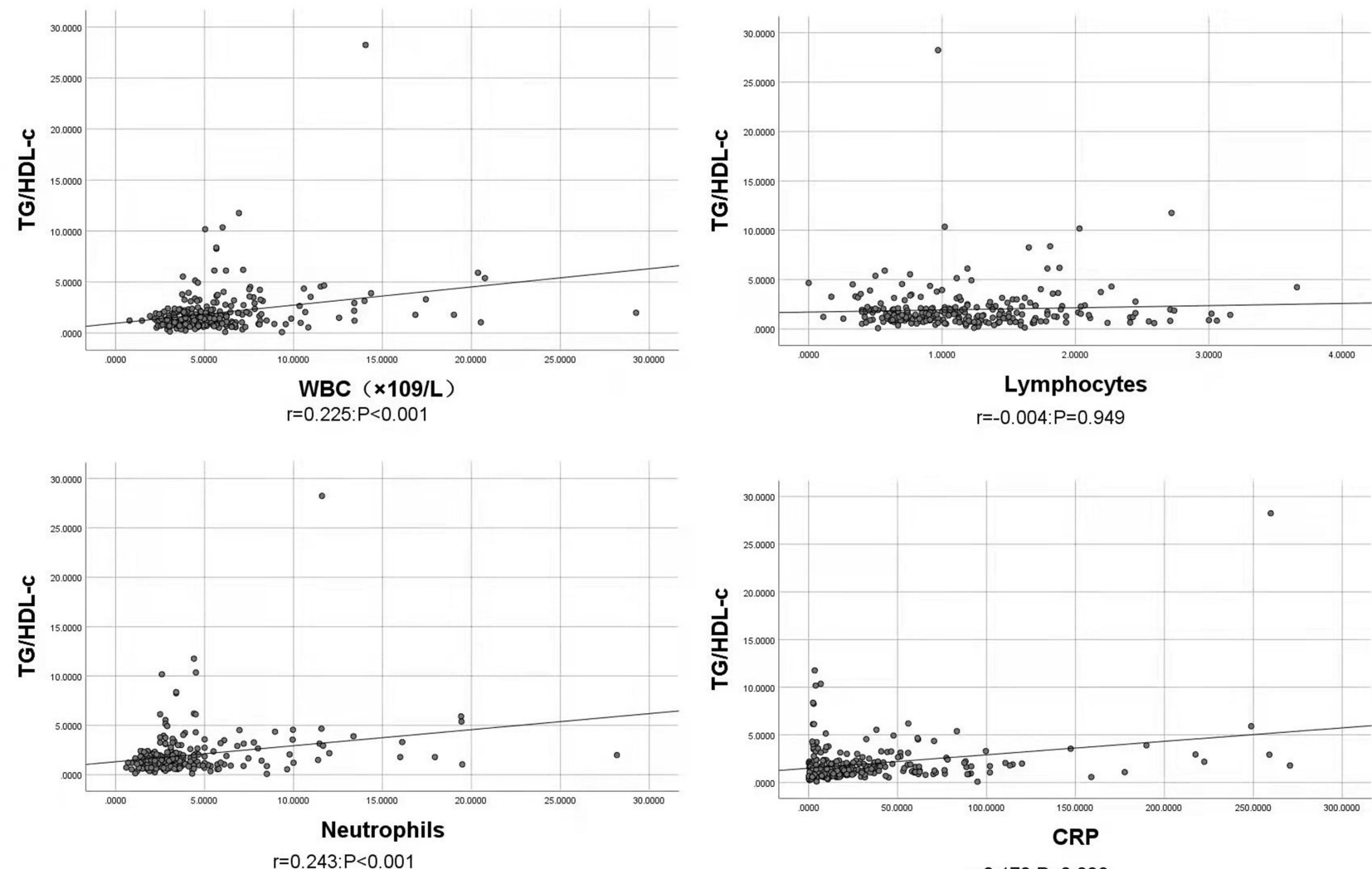

$r=0.170 ; P=0.006$

Figure 3 Association between TG/HDL-c ratio and inflammatory indicators. TG/HDL-c ratio positively correlated with WBC $(r=0.255, P<0.00 \mathrm{I})$, neutrophils $(r=0.243$, $P<0.00 \mathrm{I})$ and $\operatorname{CRP}(r=0.170, P<0.006)$, but no significant correlations existed between TG/HDL-c ratio and lymphocytes $(r=-0.004, P=0.949)$.

hypertriglyceridemia eventually. ${ }^{19}$ Hypertriglyceridemia can cause endothelial dysfunction, thus leading to a higher susceptibility to complications related to cardiovascular diseases in COVID-19 patients. ${ }^{20}$ Furthermore, TG could regulate the expression of angiotensin-converting enzyme-2 (ACE2) protein through methylenetetrahydrofolate

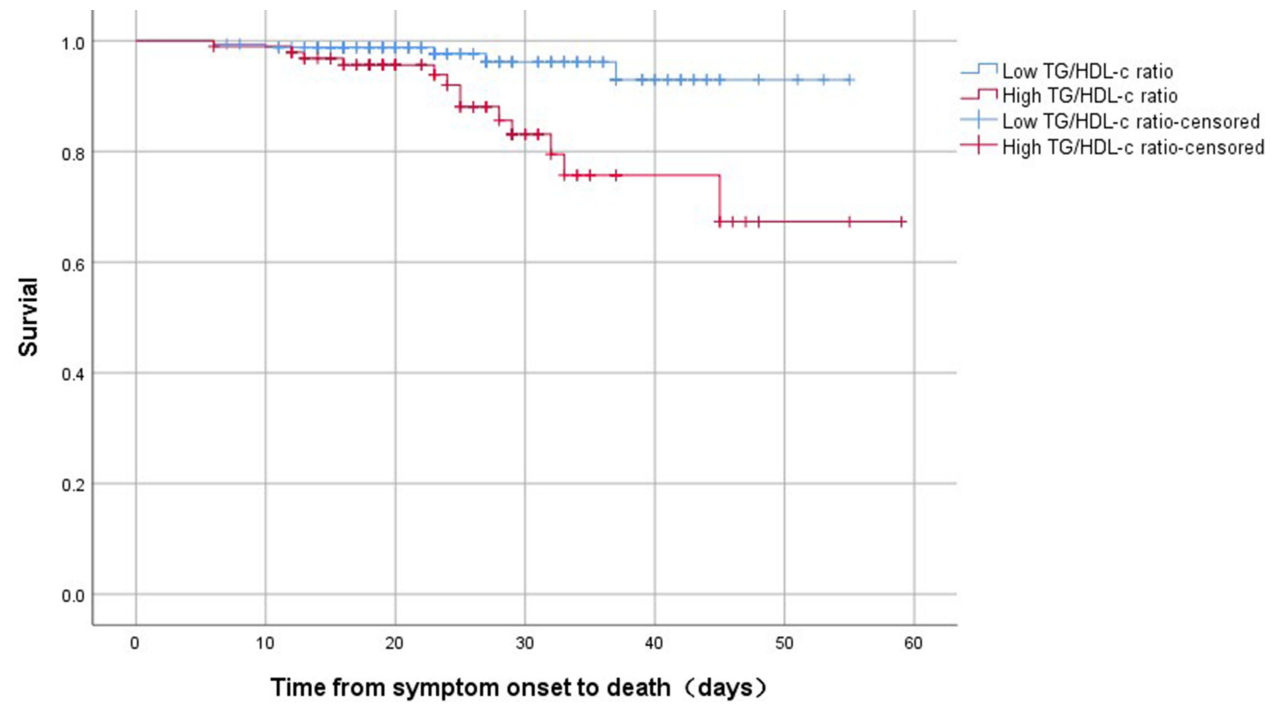

Figure 4 The time-dependent risk of death in COVID-19 patients with high and low TG/HDL-c ratio using Kaplan-Meier curve. Patients with high TG/HDL-c ratio showed a worse survival compared with those with low TG/HDL-c ratio (Log rank $P=0.003)$. 
Table 5 Demographics and Baseline Characteristics of Survivor and Non-Survivor of COVID-19 Patients without Underlying Diseases

\begin{tabular}{|c|c|c|c|c|}
\hline & No. $(\%)$ Total $(n=174)$ & Survivor $(n=165)$ & Non-Survivor $(n=9)$ & $P$ value \\
\hline Age, \% & & & & $<0.001$ \\
\hline$\geq 65 y$ & $20(11.5)$ & $16(9.7)$ & $4(44.4)$ & \\
\hline $45 \leq$ age $<65$ & $60(34.5)$ & $55(33.3)$ & $5(55.6)$ & \\
\hline$<45 y$ & $94(54.0)$ & $94(57.0)$ & $0(0.0)$ & \\
\hline \multicolumn{5}{|l|}{ Gender, \% } \\
\hline Male & $88(52.3)$ & $84(50.9)$ & $4(44.4)$ & 0.706 \\
\hline Female & $86(47.7)$ & $81(49.1)$ & $5(55.6)$ & \\
\hline \multicolumn{5}{|l|}{ Symptoms } \\
\hline Fever, \% & $116(66.7)$ & $108(65.5)$ & $8(88.9)$ & 0.148 \\
\hline Cough, \% & I47 (84.5) & I $40(84.8)$ & $7(77.8)$ & 0.608 \\
\hline Myalgia, \% & $16(9.2)$ & $16(9.7)$ & $0(0.0)$ & 0.328 \\
\hline Fatigue, \% & $74(42.5)$ & $72(43.6)$ & $2(22.2)$ & 0.207 \\
\hline Headache, \% & $23(13.2)$ & $23(13.9)$ & $0(0.0)$ & 0.231 \\
\hline Diarrhoea, \% & $43(24.7)$ & $41(24.8)$ & $2(22.2)$ & 0.859 \\
\hline Abdominal pain, \% & $3(1.7)$ & $3(1.8)$ & $0(0.0)$ & 0.684 \\
\hline Shortness of breath, \% & $53(30.5)$ & $50(30.3)$ & $3(33.3)$ & 0.848 \\
\hline
\end{tabular}

Notes: $P$ values indicate differences between survivor and non-survivor of COVID-19 patients without underlying diseases. $P<0.05$ was considered statistically significant (marked in bold).

Abbreviation: COVID-19, Coronavirus disease 2019.

dehydrogenase (MTHFD1) which affected the methylation of the ACE2. ${ }^{21}$ Meanwhile, it was reported that TG was associated with the excessive activation of macrophages, ${ }^{22}$ positive with the levels of CRP and procalcitonin, ${ }^{23}$ and the level was significantly increased in COVID-19 patients with poor prognoses. ${ }^{24}$

The most striking function of HDL is to facilitate reverse cholesterol transport from tissues to the liver. ${ }^{25} \mathrm{HDL}$ particles are crucial for the immune system and defense against infectious diseases, which can mitigate inflammatory responses during sepsis, ${ }^{26,27}$ and function against RNA and DNA viruses. ${ }^{28}$ In addition, HDL has the greatest affinity for binding and neutralizing lipopolysaccharides and lipoteichoic acid, ${ }^{29}$ and also exerts antithrombotic ${ }^{30}$ and antioxidant effects. ${ }^{31}$ Using genetic variants as risk factors, a prior research identified that genetically determined increased levels of HDL-c exhibited an association with reduced mortality from sepsis. ${ }^{16}$ At the same time, it was suggested that a genetic variant in cholesteryl ester transfer protein (CETP), rs1800777, was related to the level of HDL-c in septic patients, ${ }^{32}$ and CETP inhibitor might be a potential therapy for sepsis. ${ }^{33}$ Some viral infections causing inflammation also resulted in dyslipidemia, in which HIV patients had a decreased HDL-c levels, ${ }^{34,35}$ and patients with hepatitis B in the cirrhosis phase showed lower HDL-c levels. ${ }^{36}$ Recently, it has been reported that COVID-19 patients with declined HDL-c

Table 6 Comparison of Laboratory Parameters Between the Survivor and Non-Survivor of COVID19 Patients without Underlying Diseases

\begin{tabular}{|l|l|l|l|}
\hline & Survivor & Non-Survivor & P value \\
\hline WBC, $\times 10^{9} / \mathrm{L}$ & $4.6(3.6-5.8)$ & $12.6(5.5-17.9)$ & $<0.00 I$ \\
Lymphocytes, $\times 10^{9} / \mathrm{L}$ & $1.2(0.9-1.7)$ & $0.6(0.4-0.7)$ & $<0.001$ \\
Neutrophils, $\times 10^{9} / \mathrm{L}$ & $2.9(2.1-3.6)$ & $11.4(4.7-17.0)$ & $<0.00 \mathrm{I}$ \\
$\mathrm{CRP}, \mathrm{mg} / \mathrm{L}$ & $12.7(4.0-27.9)$ & $70.1(29.6-254.3)$ & $<0.00 \mathrm{I}$ \\
TC/HDL-c ratio & $4.5(3.7-5.6)$ & $4.9(3.3-5.5)$ & 0.899 \\
LDL-c/HDL-c ratio & $3.2(2.5-4.1)$ & $2.2(1.5-3.5)$ & $\mathbf{0 . 0 1 0}$ \\
TG/HDL-c ratio & $1.3(0.9-2.0)$ & $1.9(1.6-8.4)$ & $\mathbf{0 . 0 0 I}$ \\
\hline
\end{tabular}

Notes: $P$ values indicate differences between survivor and non-survivor of COVID-19 patients without underlying diseases. $P<0.05$ was considered statistically significant (marked in bold).

Abbreviations: COVID-19, Coronavirus disease 2019; WBC, White blood cells; CRP, C-reactive proteins; TC/HDL-c ratio, total cholesterol-to-high-density lipoprotein cholesterol ratio; LDL-c/HDL-c ratio, low-density lipoprotein cholesterol-to-high-density lipoprotein cholesterol ratio; TG/HDL-c ratio, triglyceride-to-high-density lipoprotein cholesterol ratio. 
Table 7 Univariate and Multivariate Analysis of Risk Factors Related to the Mortality of COVID-19 Patients without Underlying Diseases

\begin{tabular}{|l|l|l|l|l|}
\hline \multirow{2}{*}{ Variables } & Univariate & \multicolumn{2}{l|}{ Multivariate } & \multicolumn{2}{l|}{ P value } \\
\cline { 2 - 5 } & P value & OR & $\mathbf{9 5 \%} \mathbf{~ I ~}$ & 0.624 \\
WBC & $<0.001$ & 0.502 & $0.032-7.881$ & 0.130 \\
Lymphocytes & $\mathbf{0 . 0 0 1}$ & 0.008 & $0.000-4.208$ & 0.438 \\
Neutrophils & $<\mathbf{0 . 0 0 1}$ & 3.319 & $0.174-56.530$ & 0.959 \\
CRP & $\mathbf{0 . 0 0 2}$ & 1.001 & $0.965-1.038$ & $\mathbf{0 . 0 2 0}$ \\
TG/HDL-c ratio & $\mathbf{0 . 0 0 8}$ & 1.744 & $1.091-2.786$ & 1 \\
LDL-c/HDL-c ratio & 0.112 & 1 & 1 & 1 \\
TC/HDL-c ratio & 0.700 & 1 & 1 & \\
\hline
\end{tabular}

Notes: $P$ values indicate differences between survivor and non-survivor of COVID-19 patients without underlying diseases. $P<0.05$ was considered statistically significant (marked in bold).

Abbreviations: COVID-19, Coronavirus disease 2019; OR, odds ratio; Cl, confidence interval; WBC, White blood cells; CRP, C-reactive proteins; TC/HDL-c ratio, total cholesterol-to-high-density lipoprotein cholesterol ratio; LDL-c/HDL-c ratio, low-density lipoprotein cholesterol-to-high-density lipoprotein cholesterol ratio; TG/HDL-c ratio, triglyceride-to-high-density lipoprotein cholesterol ratio.

concentrations had longer time for viral nucleic acid amplification test turning negative than those with normal levels, ${ }^{37}$ and lower HDL-c levels exhibited an association with the severity of COVID-19 in patients. ${ }^{38}$ In view of the above, it has been well documented that there may be a marked decrease in HDL-c concentrations during the acute phase response, however, the mechanisms underlying this decrease are not clearly defined. Apolipoprotein A1 (ApoA-1), a major structural protein of HDL-c, was reported to be decreased together with lower HDL-c when pro-inflammatory cytokines (eg, IL-6 and CRP) inhibited the activity of apolipoprotein synthesis enzymes. ${ }^{24,27}$ Serum amyloid A (SAA)-enriched HDL displaced and decreased ApoA-1 levels, and scavenged HDL more rapidly, which was significantly higher in patients diagnosed with severe COVID-19. ${ }^{17,24}$ Paraoxonase 1 (PON1), an antioxidant enzyme of HDL, could be inactivated under oxidative stress and further weaken HDL functions. ${ }^{39}$ Moreover, hemodilution, consumption of HDL particles, and capillary leaks could also explain the decreased HDL concentration, all of which might be applicable for COVID-19 patients. ${ }^{27,40}$ In addition, impaired antioxidant properties of HDL could cause lipid oxidation, hence inducing inflammation and accentuating tissue damage. ${ }^{20}$ Consequently, HDL-c deficiency can induce cytokine overproduction, and these overproduced cytokines can in turn prime the depletion of HDL-c, thus promoting a vicious cycle in severe patients. Collectively, systemic inflammatory responses can cause hypertriglyceridemia and decrease HDL-c, resulting in an increase in TG/HDL-c ratio.

Inflammatory cells can accelerate the release of various cytokines in the pathophysiological process during SARS-CoV-2 infection, thus leading to a cytokine storm that may induce rapid development in multiple organ dysfunctions or even death. ${ }^{41}$ Abundant evidence in the past has indicated that there was a strong association of compromised immune functions and excessive inflammatory response with mortality from COVID-19. ${ }^{42-44}$ In the current study, the TG/HDL-c ratio was positively related to the levels of $\mathrm{WBC}$, neutrophils, and CRP. These findings were similar to the study mentioned that IPAH patients with elevated TG/HDL-c ratio had elevated levels of IL-1 $\beta$, MCP-1, and IL- $6 .{ }^{10}$ In our further analysis, a ROC curve and KM curve were generated with the discovery that TG/HDL-c ratio was probably an alternative prognostic predictor for COVID-19. Based on the findings presented above, it can be speculated that inflammation monitoring might be useful in predicting the mortality of COVID-19 patients who had elevated TG/HDL-c ratio. Anyway, further investigations are required for the elaboration of specific mechanisms of TG/HDL-c ratio on COVID-19.

To date, effective pharmacological interventions still have not been identified for the treatment of COVID- 19 . Repurposed drugs have the advantages of widely acknowledged pharmacokinetics, pharmacodynamics, adverse effects, route of administration, and dosing regimens, which has become a promising approach in the treatment of COVID$19{ }^{45,46}$ A series of randomized controlled trials (RCTs) have been conducted to explore the clinical efficacy of lipidmodulating agents on COVID-19, and has revealed that they may offer therapeutic potential in COVID-19. ${ }^{47}$ Omega-3 polyunsaturated fatty acids (PUFAs), stains and fibrates are expected to ameliorate dyslipidemia by lowering TG and 
raising HDL. ${ }^{48-52}$ Moreover, HDL-increasing drugs, such as cholesterol acyltransferase (LCAT) and Niacin, have also been studied as potential therapeutic strategies for COVID-19 treatment. ${ }^{47,49}$

The study still has some limitations. First, due to the retrospective analysis, relevant variables (eg, BMI) were not identified in our study and the small sample size of survivors, which might cause bias. Next, the time patients having lipids tests was in different phase of COVID-19 infection for the reason of earlier or later onset of symptoms to enter the hospital which could also make a bias. Third, there is not a public database providing biomarkers before patients suffered diseases, which might make the research more convincing. Fourth, there was no further detection of TG and HDL-c levels in patients during hospitalization. Dynamic monitoring might be a better characterization for dyslipidemia. Finally, lipid metabolism can be affected by various factors, such as dietary preferences and habits, and the mechanisms should be further studied.

\section{Conclusion}

To sum up, our study suggested that higher TG/HDL-c ratio might benefit the identification of COVID-19 patients who had a high likelihood of developing a low survival. Therefore, rigorous control of lipid parameters is essential during COVID-19 pandemic, and treatment with TG-lowering or HDL-raising agents may improve the prognosis of COVID-19. Large sample, multi-center prospective studies and pathophysiological mechanisms related lipids and COVID-19 should be performed in the future.

\section{Abbreviations}

TG/HDL-c, triglyceride to high density lipoprotein cholesterol ratio; COVID-19, Coronavirus disease 2019; SARS-CoV-2, severe acute respiratory syndrome coronavirus 2; TG, triglyceride; HDL-c, high density lipoprotein cholesterol; IPAH, idiopathic pulmonary arterial hypertension; AAD, acute aortic dissection; ROC, receiver operating characteristics; WBC, white blood cells; CRP, C-reactive proteins; TC/HDL-c ratio, total cholesterol-to-high-density lipoprotein cholesterol ratio; LDL-c/HDL-c ratio, low-density lipoprotein cholesterol-to-high-density lipoprotein cholesterol ratio; OR, odds ratio; 95\% CI, confidence interval; AUC, area under the curve; TNF, tumor necrosis factor; ACE2, angiotensin-converting enzyme-2; MTHFD1, methylenetetrahydrofolate dehydrogenase; CETP, cholesteryl ester transfer protein; ApoA-1, Apolipoprotein-1; IL-6, interleukin-6; SAA, Serum amyloid A; PON1, Paraoxonase 1; HIV, human immunodeficiency virus; RCTs, randomized controlled trials; PUFAs, polyunsaturated fatty acids; LCAT, cholesterol acyltransferase.

\section{Data Sharing Statement}

The data presented in this study are available on request from the corresponding author Yanjun Zhong on reasonable request.

\section{Ethics Statement}

The study was approved by the Ethics Committee of Second Xiangya Hospital of Central South University (No.2020001). Patient consent was waived due to the retrospective study and there was no intervention during the study.Furthermore, patient data confidentiality are maintained in compliance with the Declaration of Helsinki.

\section{Author Contributions}

All authors made a significant contribution to the work reported, whether that is in the conception, study design, execution, acquisition of data, analysis and interpretation, or in all these areas; took part in drafting, revising or critically reviewing the article; gave final approval of the version to be published; have agreed on the journal to which the article has been submitted; and agreed to be accountable for all aspects of the work.

\section{Funding}

This study was funded by Emergency Project of Prevention and Control for COVID-19 of Central South University: 160260005, and the Natural Science Foundation of Hunan Province, China: 2021JJ40896. 


\section{Disclosure}

The authors declare that the research was conducted in the absence of any commercial or financial relationships that could be construed as a potential conflict of interest.

\section{References}

1. Wu Z, McGoogan JM. Characteristics of and important lessons from the coronavirus disease 2019 (COVID-19) outbreak in China: summary of a report of 72314 cases from the Chinese Center for Disease Control and Prevention. JAMA. 2020;323(13):1239-1242. doi:10.1001/ jama.2020.2648

2. Zhou F, Yu T, Du R, et al. Clinical course and risk factors for mortality of adult inpatients with COVID-19 in Wuhan, China: a retrospective cohort study. Lancet. 2020;395(10229):1054-1062. doi:10.1016/S0140-6736(20)30566-3

3. Cordero A, Laclaustra M, León M, et al. Comparison of serum lipid values in subjects with and without the metabolic syndrome. Am J Cardiol. 2008;102(4):424-428. doi:10.1016/j.amjcard.2008.03.079

4. Prasad M, Sara J, Widmer RJ, Lennon R, Lerman LO, Lerman A. Triglyceride and triglyceride/HDL (high density lipoprotein) ratio predict major adverse cardiovascular outcomes in women with non-obstructive coronary artery disease. J Am Heart Assoc. 2019;8(9):e009442. doi:10.1161/ JAHA.118.009442

5. Wang X, Dong Y, Qi X, Huang C, Hou L. Cholesterol levels and risk of hemorrhagic stroke: a systematic review and meta-analysis. Stroke. 2013;44(7):1833-1839. doi:10.1161/STROKEAHA.113.001326

6. Zavaroni I, Bonora E, Pagliara M, et al. Risk factors for coronary artery disease in healthy persons with hyperinsulinemia and normal glucose tolerance. $N$ Engl J Med. 1989;320(11):702-706. doi:10.1056/NEJM198903163201105

7. Le Lay S, Simard G, Martinez MC, Andriantsitohaina R. Oxidative stress and metabolic pathologies: from an adipocentric point of view. Oxid Med Cell Longev. 2014;2014:908539. doi:10.1155/2014/908539

8. Krawczyk M, Rumińska M, Witkowska-Sędek E, Majcher A, Pyrżak B. Usefulness of the triglycerides to high-density lipoprotein cholesterol ratio (TG/HDL-C) in prediction of metabolic syndrome in polish obese children and adolescents. Acta Biochim Pol. 2018;65(4):605-611. doi:10.18388/ abp.2018_2649

9. Klisic A, Kavaric N, Ninic A. Serum cystatin C levels are associated with triglycerides/high-density lipoprotein cholesterol ratio in adolescent girls ages between 16-19 years old. Eur Rev Med Pharmacol Sci. 2020;24(20):10680-10686. doi:10.26355/eurrev_202010_23426

10. Jonas K, Magoń W, Podolec P, Kopeć G. Triglyceride-to-high-density lipoprotein cholesterol ratio and systemic inflammation in patients with idiopathic pulmonary arterial hypertension. Med Sci Monit. 2019;25:746-753. doi:10.12659/MSM.912766

11. Zhang B, Dong C, Li S, Song X, Wei W, Liu L. Triglyceride to high-density lipoprotein cholesterol ratio is an important determinant of cardiovascular risk and poor prognosis in coronavirus disease-19: a retrospective case series study. Diabetes Metab Syndr Obes. 2020;13:3925-3936. doi:10.2147/DMSO.S268992

12. Masana L, Correig E, Ibarretxe D, et al. Low HDL and high triglycerides predict COVID-19 severity. Sci Rep. 2021;11(1):7217. doi:10.1038/ s41598-021-86747-5

13. Alcántara-Alonso E, Molinar-Ramos F, González-López JA, et al. High triglyceride to HDL-cholesterol ratio as a biochemical marker of severe outcomes in COVID-19 patients. Clin Nutr ESPEN. 2021;44:437-444. doi:10.1016/j.clnesp.2021.04.020

14. Peng F, Lei S, Zhang Q, Zhong Y, Wu S. Smoking is correlated with the prognosis of coronavirus disease 2019 (COVID-19) patients: an observational study. Front Physiol. 2021;12:634842. doi:10.3389/fphys.2021.634842

15. Khovidhunkit W, Kim MS, Memon RA, et al. Effects of infection and inflammation on lipid and lipoprotein metabolism: mechanisms and consequences to the host. J Lipid Res. 2004;45(7):1169-1196. doi:10.1194/jlr.R300019-JLR200

16. Trinder M, Walley KR, Boyd JH, Brunham LR. Causal inference for genetically determined levels of high-density lipoprotein cholesterol and risk of infectious disease. Arterioscler Thromb Vasc Biol. 2020;40(1):267-278. doi:10.1161/ATVBAHA.119.313381

17. Wendel M, Paul R, Heller AR. Lipoproteins in inflammation and sepsis. II. Clinical aspects. Intensive Care Med. 2007;33(1):25-35. doi:10.1007/ s00134-006-0433-X

18. Lu B, Moser A, Shigenaga JK, Grunfeld C, Feingold KR. The acute phase response stimulates the expression of angiopoietin like protein 4. Biochem Biophys Res Commun. 2010;391(4):1737-1741. doi:10.1016/j.bbrc.2009.12.145

19. Lim MA. Exercise addiction and COVID-19-associated restrictions. J Ment Health. 2021;30(2):135-137. doi:10.1080/09638237.2020.1803234

20. Sorokin AV, Karathanasis SK, Yang ZH, Freeman L, Kotani K, Remaley AT. COVID-19-associated dyslipidemia: implications for mechanism of impaired resolution and novel therapeutic approaches. FASEB J. 2020;34(8):9843-9853. doi:10.1096/fj.202001451

21. Ma X, Li X, Wan BO, Miao Z. Triglyceride regulate ACE2 level through MTHFD1. J Biosci. 2021;46(3):57. doi:10.1007/s12038-021-00178-y

22. Zhong P, Wang Z, Du Z. Serum triglyceride levels and related factors as prognostic indicators in COVID-19 patients: a retrospective study. Immun Inflamm Dis. 2021;9(3):1055-1060. doi:10.1002/iid3.469

23. Bellia A, Andreadi A, Giudice L, et al. Atherogenic dyslipidemia on admission is associated with poorer outcome in people with and without diabetes hospitalized for COVID-19. Diabetes Care. 2021;44(9):2149-2157. doi:10.2337/dc20-2838

24. Sun JT, Chen Z, Nie P, et al. Lipid profile features and their associations with disease severity and mortality in patients with COVID-19. Front Cardiovasc Med. 2020;7:584987. doi:10.3389/fcvm.2020.584987

25. Rader DJ, Alexander ET, Weibel GL, Billheimer J, Rothblat GH. The role of reverse cholesterol transport in animals and humans and relationship to atherosclerosis. J Lipid Res. 2009;50(Suppl):S189-194. doi:10.1194/jlr.R800088-JLR200

26. Jahangiri A, de Beer MC, Noffsinger V, et al. HDL remodeling during the acute phase response. Arterioscler Thromb Vasc Biol. $2009 ; 29$ (2):261-267. doi:10.1161/ATVBAHA.108.178681

27. Pirillo A, Catapano AL, Norata GD. HDL in infectious diseases and sepsis. Handb Exp Pharmacol. 2015;224:483-508.

28. Meilhac O, Tanaka S, Couret D. High-density lipoproteins are bug scavengers. Biomolecules. 2020;10(4):598. doi:10.3390/biom10040598

29. Levels JH, Marquart JA, Abraham PR, et al. Lipopolysaccharide is transferred from high-density to low-density lipoproteins by lipopolysaccharide-binding protein and phospholipid transfer protein. Infect Immun. 2005;73(4):2321-2326. doi:10.1128/IAI.73.4.2321-2326.2005 
30. Birjmohun RS, van Leuven SI, Levels JH, et al. High-density lipoprotein attenuates inflammation and coagulation response on endotoxin challenge in humans. Arterioscler Thromb Vasc Biol. 2007;27(5):1153-1158. doi:10.1161/ATVBAHA.106.136325

31. Karathanasis SK, Freeman LA, Gordon SM, Remaley AT. The changing face of HDL and the best way to measure it. Clin Chem. 2017;63 (1):196-210. doi:10.1373/clinchem.2016.257725

32. Genga KR, Trinder M, Kong HJ, et al. CETP genetic variant rs 1800777 (allele A) is associated with abnormally low HDL-C levels and increased risk of AKI during sepsis. Sci Rep. 2018;8(1):16764. doi:10.1038/s41598-018-35261-2

33. Yvan-Charvet L, Kling J, Pagler T, et al. Cholesterol efflux potential and antiinflammatory properties of high-density lipoprotein after treatment with niacin or anacetrapib. Arterioscler Thromb Vasc Biol. 2010;30(7):1430-1438. doi:10.1161/ATVBAHA.110.207142

34. Baker J, Ayenew W, Quick H, et al. High-density lipoprotein particles and markers of inflammation and thrombotic activity in patients with untreated HIV infection. J Infect Dis. 2010;201(2):285-292. doi:10.1086/649560

35. Rose H, Hoy J, Woolley I, et al. HIV infection and high density lipoprotein metabolism. Atherosclerosis. 2008;199(1):79-86. doi:10.1016/j. atherosclerosis.2007.10.018

36. Cao WJ, Wang TT, Gao YF, Wang YQ, Bao T, Zou GZ. Serum lipid metabolic derangement is associated with disease progression during chronic HBV infection. Clin Lab. 2019;65(12). doi:10.7754/Clin.Lab.2019.190525

37. Ding X, Zhang J, Liu L, et al. High-density lipoprotein cholesterol as a factor affecting virus clearance in covid-19 patients. Respir Med. 2020;175:106218. doi:10.1016/j.rmed.2020.106218

38. Wang G, Zhang Q, Zhao X, et al. Low high-density lipoprotein level is correlated with the severity of COVID-19 patients: an observational study. Lipids Health Dis. 2020;19(1):204. doi:10.1186/s12944-020-01382-9

39. Levy BD, Kohli P, Gotlinger K, et al. Protectin D1 is generated in asthma and dampens airway inflammation and hyperresponsiveness. $J$ Immunol. 2007;178(1):496-502. doi:10.4049/jimmunol.178.1.496

40. Tanaka S, De Tymowski C, Assadi M, et al. Lipoprotein concentrations over time in the intensive care unit COVID-19 patients: results from the ApoCOVID study. PLoS One. 2020;15(9):e0239573. doi:10.1371/journal.pone.0239573

41. Chen $\mathrm{G}, \mathrm{Wu} \mathrm{D}$, Guo W, et al. Clinical and immunological features of severe and moderate coronavirus disease 2019. J Clin Invest. 2020;130 (5):2620-2629. doi:10.1172/JCI137244

42. Huang C, Wang Y, Li X, et al. Clinical features of patients infected with 2019 novel coronavirus in Wuhan, China. Lancet. 2020;395 (10223):497-506. doi:10.1016/S0140-6736(20)30183-5

43. Li X, Xu S, Yu M, et al. Risk factors for severity and mortality in adult COVID-19 inpatients in Wuhan. J Allergy Clin Immunol. 2020;146 (1):110-118. doi:10.1016/j.jaci.2020.04.006

44. Verity R, Okell LC, Dorigatti I, et al. Estimates of the severity of coronavirus disease 2019: a model-based analysis. Lancet Infect Dis. 2020;20 (6):669-677. doi:10.1016/S1473-3099(20)30243-7

45. Pushpakom S, Iorio F, Eyers PA, et al. Drug repurposing: progress, challenges and recommendations. Nat Rev Drug Discov. 2019;18(1):41-58. doi:10.1038/nrd.2018.168

46. Cusinato J, Cau Y, Calvani AM, et al. Repurposing drugs for the management of COVID-19. Expert Opin Ther Pat. 2021;31(4):295-307. doi:10.1080/13543776.2021.1861248

47. Talasaz AH, Sadeghipour P, Aghakouchakzadeh M, et al. Investigating lipid-modulating agents for prevention or treatment of COVID-19: JACC state-of-the-art review. J Am Coll Cardiol. 2021;78(16):1635-1654. doi:10.1016/j.jacc.2021.08.021

48. Rezaei A, Neshat S, Heshmat-Ghahdarijani K. Alterations of lipid profile in COVID-19: a narrative review. Curr Probl Cardiol. $2021 ; 100907$. doi:10.1016/j.cpcardiol.2021.100907

49. Wang G, Deng J, Li J, et al. The role of high-density lipoprotein in COVID-19. Front Pharmacol. 2021;12:720283. doi:10.3389/fphar.2021.720283

50. Yanai $\mathrm{H}$, Masui Y, Katsuyama $\mathrm{H}$, et al. An improvement of cardiovascular risk factors by omega-3 polyunsaturated fatty acids. $J$ Clin Med Res. 2018;10(4):281-289. doi:10.14740/jocmr3362w

51. Surma S, Banach M, Lewek J. COVID-19 and lipids. the role of lipid disorders and statin use in the prognosis of patients with SARS-CoV-2 infection. Lipids Health Dis. 2021;20(1):141. doi:10.1186/s12944-021-01563-0

52. Fijen LM, Grefhorst A, Levels JHM, et al. Severe acquired hypertriglyceridemia following COVID-19. BMJ Case Rep. 2021;14(11):e246698. doi:10.1136/bcr-2021-246698

International Journal of General Medicine

Dovepress

\section{Publish your work in this journal}

The International Journal of General Medicine is an international, peer-reviewed open-access journal that focuses on general and internal medicine, pathogenesis, epidemiology, diagnosis, monitoring and treatment protocols. The journal is characterized by the rapid reporting of reviews, original research and clinical studies across all disease areas. The manuscript management system is completely online and includes a very quick and fair peer-review system, which is all easy to use. Visit http://www.dovepress.com/testimonials.php to read real quotes from published authors.

Submit your manuscript here: https://www.dovepress.com/international-journal-of-general-medicine-journal 